\title{
Nest-building Behaviour of the Asian Open Billed Stork Anastomus oscitans, in the Kulik Bird Sanctuary, Raiganj, India
}

\author{
A.K. Pramanik ${ }^{1}$, K.B. Santra ${ }^{2 *}$ and C.K. Manna ${ }^{1}$ \\ ${ }^{1}$ Endocrinology Laboratory, Department of Zoology, University of Kalyani, Kalyani- 741235, West Bengal, \\ India \\ ${ }^{2}$ Department of Zoology, Daulatpur High School, Daulatpur, Dakshin Dinajpur, West Bengal, India \\ *E-mail: kalyan_117@rediffmail.com
}

Received: 20.03.2009, Accepted: 18.12.2009

\begin{abstract}
A field study of the nest-building behaviour in the breeding season of the Asian Open-Billed Stork, Anastomus oscitans, was conducted in the Kulik Bird Sanctuary, Raiganj, Uttar Dinajpur, West Bengal, India. Observations were made throughout one complete breeding season (2007-2008) in the sanctuary. The open-billed stork comes in the last week of June at the Kulik bird sanctuary, stays there for 5 to 6 months and leaves the place in the month of December of every year. From the present observations it was noticed that about 58920 storks came to Kulik during 2008. Various types of behaviour were observed during this particular period. Coming to the Kulik, pairing of the individual storks start and mating display was observed within a day or two. Birds of a pair supplied the nest building materials to form the nest. To give a definite shape to the nest it took about 12-15 days before egg laying. The nesting territory consisted of a semicircle, some times circular and approximately with $30.21 \pm 0.12 \mathrm{~cm}$ radius. The depth of the nest was about $6.9 \pm 0.02 \mathrm{~cm}$. The nest building materials consisted of fragments of old branches of trees, some soft green leaves and grasses. These branches helped in strengthening and the leaves and grasses helped in softening of the nest. During the incubation period both sexes were involved to incubate the eggs. Nest attendance of the breeding birds was continuous during the first 2-months period. From regular observation it was noticed that either of the pair took additional care by spreading their wings during the time of heavy sunshine or heavy rain. The main behavioral patterns examined were aerial displays, mating pattern, nest building, egg laying and incubation pattern, nest defense and nest protection. Diversity of nesting behavior in openbilled stork was pointed out and discussed.
\end{abstract}

Key words: As ian Open-Billed Stork, Kulik Bird Sanctuary, Nest-build ing behavior

\section{Introduction}

The Asian Open-Billed Stork, Anastomus oscitans, is a resident colonial breeder distributed in tropical Southeast Asia. These birds usually move to the lower part of the Himalayan ranges and spend about six months for the purpose of breeding. The adults are white with black wing flight feathers, red legs and dull yellow-gray bill.
The mandibles do not meet except at the tip, and this gives rise to the species name i.e., Open Billed. Non-breeding adults have the white of the plumage replaced by off-white color. Young have brown tinge to the plumage.

At the Kulik bird sanctuary, this stork species, Anastomus oscitans, breeds 
A.K. Pramanik, K.B. Santra and C.K. Manna / Our Nature (2009) 7: 39-47

together with five other members of the order- Ciconiiformes (Datta and Pal, 1993). Nest building behaviour is often associated with courtship and pair formation in birds. The degree to which this behaviour is used in courtship varies from mere manipulation of a piece of nest material or display of a potential nest site to the building of an entire nest by the male individual (Collias and Collias, 1984). Nest building behaviour may signal the reproductive performance of individuals and physiologically stimulate a partner (Collias, 1964), but there is very scanty information on the importance of the nest itself and its role in mate choice (Hoi et $a l .$, 1994). The nest may indicate parental quality, experience or genetic quality and females could therefore benefit from mating with a superior nest builder. In a comparative study of nest size in relation to parental effort in birds showed that bird species in which both sexes build their nest have larger nests than those in which only the female builds (Soler et al., 1998). Several studies have shown that the nest building ability of males is related to female mate choice and the reproductive success of the pair (Hoi et al., 1994, 1996). Nest size was positively correlated with the amount of parental investment among passerines. Thus Soler et al., (1998) concluded that nest size could indicate the willingness of males to invest in reproduction and it could therefore be a post mating sexually selected trait.

The ecology of this species in Kulik bird sanctuary, Raiganj, India has been described in few studies focusing on its population status, feeding habits as well as its conservation (Datta, 1992) but relevant informations are completely lacking in the areas of nest-building behaviour and reproduction of the Asian open-billed stork. In the present study we present some relevant data on nest building behaviour and reproductive activities of the open-billed stork in Kulik bird sanctuary to examine and determine the nesting site characteristics, tree preferences, nest numbers and arrival times, to breeding of the open-billed stork during the entire reproductive cycle. An attempt has also been made to analyze the relationship between the male's contributions to nest building and also the relationship between female's quality and investment in reproduction as per the field condition.

\section{Materials and methods}

This study was conducted in the Kulik bird sanctuary, Raiganj $\left(25^{\circ} 37^{\prime} \mathrm{N}\right.$ and $88^{\circ} 07^{\prime} \mathrm{E}$ to $25^{\circ} 62^{\prime} \mathrm{N}$ and $88^{\circ} 12^{\top} \mathrm{N}$ ), West Bengal, India in the year 2008. The area of the sanctuary is around $1.30 \mathrm{~km}^{2}$. The core area is about $0.14 \mathrm{~km}^{2}$ and the rest is the buffer area. The sanctuary has a thick canopy of broadleaved deciduous trees, which provide the nesting place of the open billed stork. The river Kulik, flows round a part of the sanctuary and acts as the boundary in its eastern and southern parts. The sanctuary is " $U$ " shaped. The sanctuary has a network of artificial canals connected with the river Kulik. Annual precipitation range varies from $1,200 \mathrm{~mm}$ to $1,550 \mathrm{~mm}$, mean winter temperature from $09^{\circ} \mathrm{C}$ to $23^{\circ} \mathrm{C}$ and summer temperature from $21^{\circ} \mathrm{C}$ to $25^{\circ} \mathrm{C}$, and humidity 75 , were recorded. During monsoon period, the river enters the sanctuary, which supports a wide variety of food for the open billed stork. The main diet of the bird is apple snail, Pila globosa or other types of snail which grows in large number in the smaller or larger water bodies surrounding Kulik.

The observations were made at daytime (06:00-18:00 hr) during the month of June 
A.K. Pramanik, K.B. Santra and C.K. Manna / Our Nature (2009) 7: 39-47

to December of the year 2008. All types of activities like aerial display, copulation, transfer of nesting materials, construction of the nest, nest defense and nest protection of the open billed stork were recoded using 10x50 binocular and a 30x telescope and relevant photographs were taken by Kodak easy share camera (C713). Copulations were regarded as successful when cloacal contact was recorded (Gonzalez-Solis and Becker, 2002). The open billed storks are sexually monomorphic and sexes are verified by their position during copulation.

All values are reported with standard error of the mean.

\section{Results}

After coming to the bird Sanctuary, the various types of behaviors especially the reproductive behaviors were recorded at the Kulik, Raiganj, W.B. in the last week of June. The birds usually fly close next to each other. Many times the stork tried to fly one above the other for a short distance. In this way the pairing of the birds occurs. Mated individuals occupied a branch of a tree and resting there side by side for a few hours (Figure 1a). The birds used to peck each other's face or head. Then aggressive behavior of both the male and female storks was observed and continued this behavior throughout the breeding season.

Copulation attempts were observed for few seconds mainly on the branch of the trees and successful cloacal contact (Figure 1b) was also observed. During this time male stood on the back of the female stork and touched the bill on the neck of the female. The frequency of the coputatory behaviour was more in the morning (10.00$12.00 \mathrm{hr})$ than in the afternoon $(02.00-04.00$ $\mathrm{hr}$ ).
Throughout the whole reproductive cycle, the construction of the nest was most conspicuous during pre-laying period. Small branches of 12 plant species (Ficus bengalensis, Anthocephalus indicus, Barringtonia acurangula, Ficus glomerata, Terminalia arjuna, Dalbergia sisoo, Bambusa tulda, Bombax ceiba, Alstonia scholaris, Streblus asper, Lagerstoemia speciosa, Trewia nudiflora) were used by the storks to construct the nest (Table 1). Among these plants species maximum number of nests were built on the tree, Lagerstoemia speciosa (Figure 2). The initiation of nest building took place in the first week of July with the first observation made approximately 15 days prior to egg laying. Most of the nest construction took place during the morning and midday hours (09.00-02.00 hr). Peak activity of nest construction was recorded between 10.00$12.00 \mathrm{hr}$.

Both the partners of a pair carried the nesting materials together during the initial stage of nest construction. Sometimes they failed to keep these nesting materials properly. Males contributed more to find and fetch the materials, while the females took charge of manipulation at the nest. More significantly the male collected the branches (Figure 1c) and other leafy materials, grass, always carried in his bill, and laid them down at the nesting site. Both the partners of a pair remained together for some hours to arrange the materials systematically for constructing the nest (Figure 1d). After making the nest base, the female individual attended the nest's ledge (Figure 1e) while the male brought branches of various trees and greeneries. Males returned at the nest with nesting materials after $50.26 \pm 0.51$ minute intervals and 
A.K. Pramanik, K.B. Santra and C.K. Manna / Our Nature (2009) 7: 39-47
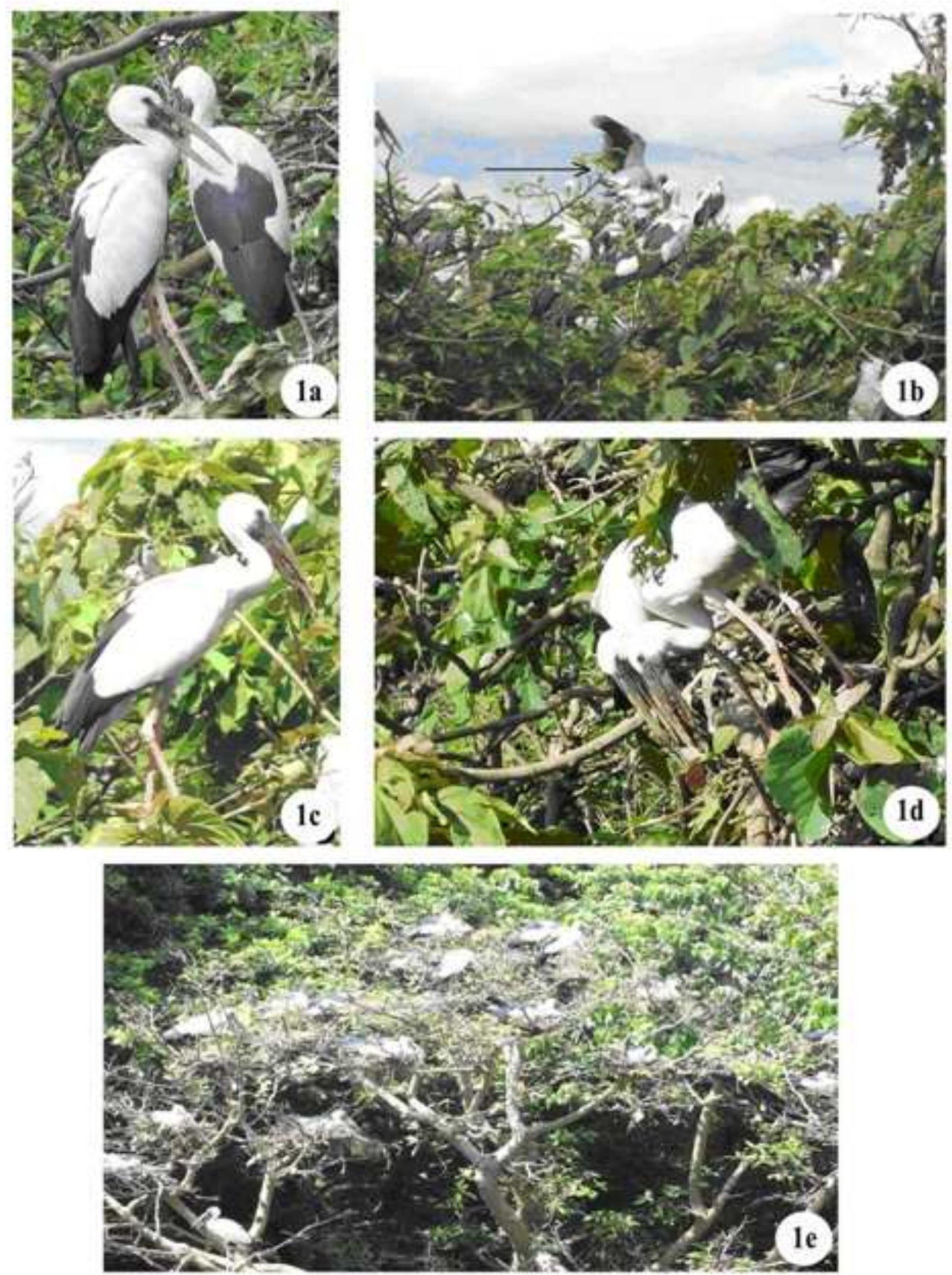

Figures: 1(a) Mated open billed storks occupy a branch of tree and resting there side by side, (b) Successful cloacal contact occurred during copulation when male stork stood on the back of the female stork on the tree, (c) The male stork collecting the branches of tree, (d) The male stork remains with the female to arrange the materials in the nest, (e) The female stork incubating the eggs and standing on the nest. 


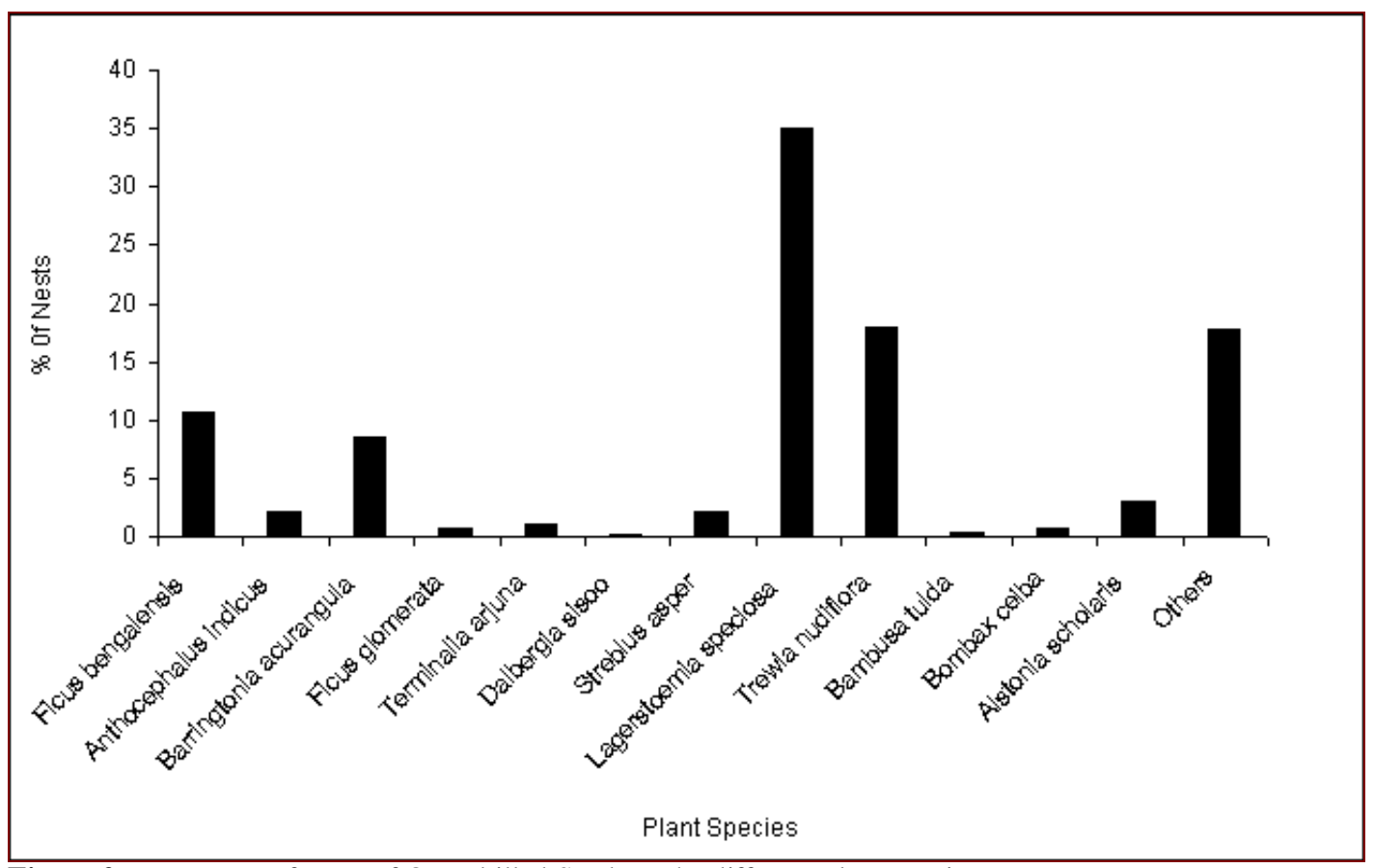

Figure 2. Percentage of nests of Open-billed Stork on the different plant species.

stayed a few minutes on the nest and went again to collect the next ones. Nest materials consisted of dry or wet and green branches, sticks and dry or green grasses. It was possible to identify 8 plant species among the constructing materials brought to the nest (Table 2). Fresh leaves of Dumur (Ficus glomerata), Eucalyptus (Eucalyptus citriodora), Jarul (Lagerstoemia speciosa), Kadamba (Anthocephalus indicus), Durba Grass (Cynodon dactylon) and Mutha Grass (Cyperus rotundus) were used more commonly in nest formation. The majority of nest building materials were collected from the vicinity of the colony at distances ranging from a few meters up to 1 to $1.5 \mathrm{~km}$ from the nests. About 11784 nests in that sanctuary were built by the open billed storks in the year 2008. Total number of nests per tree, length of the nest, breadth and depth of the nests were counted, measured and recorded in the table (Table 3).

The female laid 2 to 5 eggs in one reproductive cycle. Average number of eggs laid per female in the nest during breeding season was 3 . Initially the colour of the egg was white and after 7days it was transformed into brownish colour. Radius, length and weight of the eggs are presented in the table (Table 3). It was also observed at the time of nest construction but before egg laying period that they took rest, sometimes they touched each other by their bill, pierced their own feathers by their bill, flapped their wings in the standing position on the constructing nest. After egg laying, nest enlargement and incubation of eggs were going on simultaneously. The female manipulated the supplied nesting materials by the male without hampering the eggs 
position at that time. Nest repairing was also noticed after hatching to nourish their chicks properly. Sometimes few nests fell down due to lack of improper nest site selection during nest building period or due to storm and heavy rain. After nest loss some of the birds who did not lay eggs at that time again start to build their new nest within a very short period. The open billed storks always chose the top most branches of the tree selected by them. About $98 \%$ nests were situated at the junction of two or three branches and only $2 \%$ nests were on the single thick strong branch of the trees. The height of the nest from the ground varied from 15 to $60 \mathrm{ft}$. There was a minimum of $1 \mathrm{ft}$ distance between nearest two nests. After hatching, dropping of the shell and other particles was made by both the partners. Constant care of the newly hatched chicks was noticed in all the nests of the sanctuary.

Table 1. Plant species selected for nest building by the Asian open-billed stork, Anastomus oscitans in Kulik bird sanctuary, Raiganj, India

\begin{tabular}{ll}
\hline Common name & Latin name \\
\hline Bat & Ficus bengalensis \\
Kadamba & Anthocephalus indicus \\
Hijal & Barringtonia acurangula \\
Dumur & Ficus glomerata \\
Arjun & Terminalia arjuna \\
Sisoo & Dalbergia sisoo \\
Seora & Streblus asper \\
Jarul & Lagerstoemia speciosa \\
Pituli & Trewia nudiflora \\
Chhatim & Alstonia scholaris \\
Bamboo & Bambusa tulda \\
Simul & Bombax ceiba \\
\hline
\end{tabular}

\section{Discussion}

In the present study the nest building behavior of the open billed stork was observed. During the time of homecoming many times the open billed stork try to fly above other stork at a short distance for sometime. In vulture also it is the female that usually occupies the top position and that process lasts on average 10 seconds (Mouze and Bagnolini, 1995). Nest defense was noticed normally including the assurance of a resource such as a nest site, food or a mate (Newton, 1998). In the present study no strong aggressive behaviour was observed at nest sites among storks but slight aggressiveness was noticed throughout the breeding season. After the formation of a pair, copulation attempts were observed for a few seconds mainly on the trees. After mating, the paired individuals usually took rest on one branch of tree for a few hours.

Table 2. Materials used (old dry branches, fresh branches with leaves of the trees and Grasses) for nest building by the Asian open-billed stork, Anastomus oscitans in Kulik bird sanctuary, Raiganj, India

\begin{tabular}{ll}
\hline Common name & Latin name \\
\hline Kadamba & Anthocephalus indicus \\
Hijal & Barringtonia acurangula \\
Jarul & Lagerstoemia speciosa \\
Pituli & Trewia nudiflora \\
Eucalyptus & Eucalyptus citriodora \\
Akash Moni & Acacia auriculiformis \\
Durba Grass & Cynodon dactylon \\
Mutha Grass & Cyperus rotundus \\
\hline
\end{tabular}

Table 3. Descriptive statistics of the nest and egg of the open-billed stork, Anastomus oscitans in Kulik bird sanctuary, Raiganj, India

\begin{tabular}{lcl}
\hline Variable & $\mathbf{n}$ & $\mathbf{X} \pm \mathbf{S E}$ \\
\hline Total No. of nest/tree & 150 & $113.30 \pm 0.99^{*}$ \\
Length of the nest $(\mathrm{cm})$ & 100 & $50.30 \pm 0.11$ \\
Breath of the nest $(\mathrm{cm})$ & 100 & $40.90 \pm 0.07$ \\
Depth of the nest $(\mathrm{cm})$ & 100 & $06.90 \pm 0.02$ \\
Radius of the egg $(\mathrm{cm})$ & 100 & $03.10 \pm 0.002$ \\
Length of the egg $(\mathrm{cm})$ & 100 & $06.07 \pm 0.004$ \\
Weight of the egg $(\mathrm{g})$ & 100 & $50.56 \pm 0.01$
\end{tabular}

Mean \pm SE (Standard Error of Mean), $n=$ No. of observations 
Although storks occupied and defended their prospective nest sites as early as one month prior to egg laying they initiated nest building about only 15 days before. The activity of nest construction was highly energy consuming as the birds were required to go many times to bring the materials for the nest. Most of the nest construction took place during the morning and midday hours (09.00-02.00 hr). Peak activity was recorded between 10.00-12.00 hr. Males contributed more significantly to the finding and fetching the materials, whereas the females took charge of manipulation at the nest. In addition the persistence of the males in carrying the maximum possible amount of items may constitute an indication of their genetic quality, parental ability and commitment to the forthcoming duties of breeding (Collias and Collias, 1984; Soler et al., 1998). Female investment in nest building can also be a signal of her willingness to invest in reproduction (Soler et al., 1998). Male activity in nest building is positively related to the amount of energy that the male invests in reproduction not only during nest building but also when feeding the nestlings (Moller, 1994). This type of male activity was also similar in some vulture species, observed by Margalida and Bertran (2000). Males may be constrained in their ability to provide females with both direct (parental quality) and indirect (genetic quality), proposed by Moller (1994). The females would benefit from mating with good nest builders by saving energy and maintaining good physical condition to face the cost of reproduction. Soler et al., (1998) proposed that nest building behaviour in passerines could be a sexually selected trait, and that nest size would be a signal of the willingness of the pair that built the nest to invest in reproduction. Few plant species were selected by the stork to construct the nest. All the plants were with minimum 20 $\mathrm{ft}$ in height. Among these plants species maximum number of nests were built on the tree Lagerstoemia speciosa, because these trees are branched, hard and bushy type, helps to construct the nest properly. The hard materials used for the construction of the nest rim and grasses and fresh green leaves were used to soften the nest. These plant species are quite common in the sanctuary. The addition of green material to the nest is a distinct feature among avian species (Wimberger, 1984). Many of the herbs and plant fragments that birds bring to the nest contain volatile chemical substances, which repel or even kill the ectoparasites (Gwinner et al., 2000). Anthocephalus indicus, Eucalyptus citriodora, Cynodon dactylon, Cyperus rotundus etc contain essential oils, esters which can exterminate or impede the activity of parasites, fungi, and bacteria that may occur in stork's nests as a result of their food type preferences. In the griffon's nests this type of phenomenon was noticed by Duru et al. (2003) and Tunalier et al. (2003). However, it can be assumed that they just select plant species for their softness and flexibility that are not only suitable for nest lining but also easy and consequently energetically profitable to collect (Xirouchakis and Mylonas, 2007).

Slagsvold (1989) showed experimentally that clutch size is positively related to nest capacity in passerines. In the present study it was noticed that average number of eggs laid per female in each nest during breeding season was 3. Clutch size of birds has traditionally been thought to be adjusted to the individual's capability of successfully raising the young. A spatio-temporal 
A.K. Pramanik, K.B. Santra and C.K. Manna / Our Nature (2009) 7: 39-47

variation also exists in clutch size. However, the mechanisms leading to a correlation between the environmental condition during chick rearing and the amount of eggs laid are still being debated. At this time continuous attendance at the nest by parent birds during the first two months of the chick's life is probably to protect it from extreme sunlight or rain and predators. Constant care of the birds was noticed in almost all the nests in the sanctuary. The most critical period of the reproductive cycle was from September and October when newly hatched individuals grew to the matured form. This time the parent birds foraged alternatively. At the age of 4-5 weeks onwards the chicks were able to move around the nest. At about 60 days the young could fly short distances. Both sexes help to brood, feed and water the young. Parental behavior is largely governed by the food requirement and development of plumage of the young. In the late fledging period male and female brings most food alternatively but in few cases the female spends much time in the nest. The behavior of the Asian open-billed stork in the Kulik bird sanctuary opened a new insight of parental care and diversity of nest pattern of this particular species.

\section{Acknowledgements}

Authors are very grateful to the Chief Conservator of Forests, wildlife (Headquarter), Chief Wildlife Warden, Government of West Bengal, The Divisional Forest Officer, Mr. Anil Roy, Raiganj Division and Conservator of Forests, North-West Circle, West Bengal for issuing permission to carry out the research work within the sanctuary. Thanks are also due to Dr. Nandini Saha, Department of
English, Kalyani University for thorough revision of the manuscript.

\section{References}

Collias, N.E. 1964. The evolution of nests and nestbuilding in birds. American Zoologist 4: 175-190.

Collias, N.E. and E.C. Collias 1984. Nest building and bird behaviour. Princeton University press, New Jersey.

Datta, T. 1992. Behavioural ecology of feeding, reproduction in open-billed stork (Anastomus oscitans) (Boddaert) at the Raiganj wildlife sanctuary, Raiganj, West Dinajpur, India. North Bengal University, Indial. (Ph.D. thes is)

Datta, T. and B.C. Pal 1993. The effect of human interference on the nesting of the open-bill stork Anastomus oscitans at the raiganj wild life sanctuary, India. Biological Conservation 64 (2): 149-154.

Duru, M.E., A. Cakir, S. Kordalis, H. Zengin, M. Harmandar, S. Izumi and T. Hirata 2003. Chemical composition and antifungal properties of essential oils of three Pistacia species. Fitoterapia 74(1-2): 170-176.

Gonzalez-Solis, J. and P.H. Becker 2002. Mounting frequency and number of cloacal contacts increase with age in common terns, Sterna hirundo. Joutnsl of Avian Biology 33(3): 306-310.

Gwinner, H., M. Oltrogge, L. Trost and U. Nienaber 2000. Green plants in starling nests: effects on nestlings. Animal Behaviour 59: 301-309.

Hoi, H., B. Schleicher and F. Valera 1994. Female mate choice and nest desertion in penduline tits, Remiz pendulinus, the importance of nest quality. Animal behaviour 48: 743-746.

Hoi, H., B. Schleicher and F. Valera 1996. Nest size variation and its importance for mate choice in penduline tits, Remiz pendulinus. Animal behaviour 51: 464-466.

Margalida, A. and J. Bertran 2000. Nest building behaviour of Bearded Vulture Gypaetus barbatus. Ardea 88(2): 259-264.

Moller, A.P. 1994. Sexual selection and the Barn Swallow. Oxford University Press, Oxford.

Mouze, M. and C. Bagnolini 1995. Le vol en tandem chez le vautour fauve (Gyps fulvus). Canadian Journal of Zoology 73: 2144-2153.

Newton, I. 1998. Population limitation in birds. Academic Press, London.

Slagsvold, T. 1989. Experiment on clutch size and 
A.K. Pramanik, K.B. Santra and C.K. Manna / Our Nature (2009) 7: 39-47

nest size in passerine birds. Oecologia 80: 297-302.

Soler, J.J., A.P. Moller and M. Soler 1998. Nest building, sexual selection and parental investment. Evolutionary Ecology 12: 427-441.

Tunalier, Z., N. Kirimer and K.H.C. Baser 2003.

Wood essential oils of Juniperus foetidissima Wild. Holzforschung 57: 140-144.
Wimberger, P.H. 1984. The use of green plant material in bird nests to avoid ectoparasites. Aku. 101: 615-618.

Xirouchakis, S.M. and M. Mylonas 2007. Breeding behaviour and parental care in the Griffon Vulture, Gyps fulvus, on the island of Crete (Greece). Ethology Ecology and Evolution 19: 1-26. 